\title{
A New Partial 3D Object Indexing and Retrieval Approach Combining 2D slices and Apriori Algorithm
}

\author{
I.O. Taybi1,A, T. Gadi²,A, R. Alaoui3,B,C \\ A Laboratory of Informatics, Imaging, and Modeling of Complex Systems (LIIMSC) \\ Faculty of Sciences and Techniques, Hassan 1st University, Settat, Morocco \\ B LRIT Laboratory, Faculty of Sciences, Mohammed V University, Rabat Morocco \\ C LASTIMI Laboratory, Higher School of Technology - Sale, Mohammed V University, \\ Rabat Morocco \\ 1 ORCID: 0ooo-0oo1-6782-9807, ilyass.ouazzani@gmail.com \\ 2 ORCID: 0ooo-0002-2174-5816, gtaoufiq@yahoo.fr \\ 3 ORCID: 0000-0001-9948-329X, alaoui.rach@gmail.com
}

\begin{abstract}
This paper examines the issue of $3 \mathrm{D}$ object indexing and retrieval and tries to solve this problem using partial indexing approach. The hypothesis in this context is that similar $3 \mathrm{D}$ objects will be composed of similar 2D slices. The proposed partial $3 \mathrm{D}$ object indexing and retrieval method is applicable on both complete and incomplete $3 \mathrm{D}$ objects, which is based on a similarity measuring between $2 \mathrm{D}$ slices of $3 \mathrm{D}$ objects. The main idea behind our approach is to extract an initial set of 2D slices corresponding to determined axes, and then use the Apriori algorithm to select the most representative ones, transforming the issue of shapematching between $3 \mathrm{D}$ objects into evaluating the similarities between their $2 \mathrm{D}$ slices. Experiments on the Princeton Shape Benchmark (PSB) indicate that our approach outperforms evaluated retrieval approaches.
\end{abstract}

Keywords: 3D object indexing, 3D object retrieval, Cluster validity index, Data mining, Apriori Algorithm, Association rules, Partial similarity.

\section{Introduction}

Because of the fact that 3D modelling and scanning tools are increasingly becoming more popular, as well as the gradual fame of $3 \mathrm{D}$ objects in different domains [1] [2], researchers' attention has been drawn to discovering processing techniques for these $3 \mathrm{D}$ objects. The significant motivation behind such research is to decrease the expense of modelling and processing, which can be achieved through conceiving indexing and retrieval approaches to make quick and consistent search engines. The main challenge in $3 \mathrm{D}$ object indexing and retrieval process is to accomplish a robust descriptor by extracting $3 \mathrm{D}$ objects' geometric and topological features, creating a signature that can distinguish them. The $3 \mathrm{D}$ object indexing and retrieval paradigms can be broadly divided into global based approaches, and partial based approaches.

Global based approaches are characterized by the global visual appearance of the 3D object. They have the capacity to describe the whole $3 \mathrm{D}$ object with a single vector. Nevertheless, they do not have the ability to correctly match the $3 \mathrm{D}$ objects when the disposable data for the latter is incomplete, imperfect, or corrupted. The partial based approaches can solve the issue of matching incomplete or imperfect $3 \mathrm{D}$ objects, in addition to their capability to supply a higher level description of a 3D model. In fact, partial based approaches are based on a thorough analysis of the partials of $3 \mathrm{D}$ objects. They are motivated by the idea that similar $3 \mathrm{D}$ ob- 
jects consist of similar parts, whereas matching refers to the comparison of $3 \mathrm{D}$ objects' parts, which is ordinarily realized by reducing a distance measure.

The proposed partial 3D object indexing and retrieval approach, which can be applied on both complete and incomplete $3 \mathrm{D}$ objects, is based on similarity computed between the 2D representative slices of each $3 \mathrm{D}$ object, transforming the issue of shape matching between $3 \mathrm{D}$ objects into assessing the similarities between their respective 2D slices. The proposed approach begins by a normalization stage to ensure that similar $3 \mathrm{D}$ objects will be treated the same way, and consequently, the matching 3D objects will generate similar $2 \mathrm{D}$ slices. Then, for each $3 \mathrm{D}$ object, we extract an initial set of $2 \mathrm{D}$ slices corresponding to determined axes. Afterwards, we characterize each $2 \mathrm{D}$ slice by a vector of Zernike moments. Next, we represent the $2 \mathrm{D}$ slices of each $3 \mathrm{D}$ object in a transactional database. After, we use the Apriori algorithm and association rules to select from the initial set of $2 \mathrm{D}$ slices the most representative ones. Finally, we apply our proposed metric to compare the request's representative $2 \mathrm{D}$ slices with those of the database. The present paper is organized as follows. In second section, we discuss some $3 \mathrm{D}$ objects retrieval approaches by classifying them into two categories, global and partial methods. Section three discusses the concept of Data Mining. In the fourth part, the proposed approach is introduced. Experimental results are provided in the fifth section. Finally, the sixth part presents the conclusion and our future work.

\section{Related work}

In the past decade, a great number of content-based techniques for the $3 \mathrm{D}$ object indexing and retrieval have been proposed [3]. In this section, we are discussing the $3 \mathrm{D}$ object descriptors sorted into two groups: global based approaches and partial based approaches.

\subsection{Global based approaches}

Generally speaking, global based approaches try to describe the $3 \mathrm{D}$ object in an overall way without paying attention to its components which can affect their results in some instances. Wang et al. [4] proposed a global way to represent the $3 \mathrm{D}$ object using voxels. They introduce NormalNet, a voxel-based convolutional neural network (CNN) way for 3D object representation and retrieval. The network employs normal vectors of the object surfaces as entry, manifesting stronger discriminatory capacity than binary voxels. Bouksim et al. [5] introduced a new way to train an artificial neural network (ANN) with a histogram of characteristics (Shape index, dihedral angle, and shape diameter function), which are extracted directly from the $3 \mathrm{D}$ object. After the training stage, the authors concatenate the hidden layers and used them like a descriptor in the retrieval process. Luciano et al. [6] used geodesic moments to propose a new geometric approach for $3 \mathrm{D}$ object retrieval. They adopted an unsupervised approach for learning shape descriptors using sparse autoencoders. Biasotti et al. [7] presented an approach, a search engine model for dataset exploration, which is based on multiple similarity criteria between models in its search. The combination of similarity criteria they are proposing is a user-driven navigation and similarity assessment. Furthermore, they explored $3 \mathrm{D}$ object collections with different variations of the $3 \mathrm{D}$ objects' properties in the dataset. They also interceded a combination of similarity criteria for user-driven navigation and similarity assessment. Yujuan et al. [8] based their work on the scale-invariant heat kernel signature (SIHKS), to create a non-rigid $3 \mathrm{D}$ shape classification approach using Convolutional Neural Networks (CNNs).

\subsection{Partial based approaches}

Researchers' attention has been drawn to partial indexing and retrieval $3 \mathrm{D}$ objects thanks to the development of $3 \mathrm{D}$ design tools and the wide accessibility of $3 \mathrm{D}$ scanners. This interest has been additionally intensified by the appearance of diverse application areas, for example, 
digital libraries of cultural heritage models, which demand partial 3D model indexing and retrieval abilities. In this specific circumstance, the scanned request can be noisy and rough, on the other hand, it is not clear if we can match an incomplete $3 \mathrm{D}$ object against a complete one, since there is a blank between their representations. In fact, these representations' gap complicates the extraction of a descriptor that will empower a coordination between an incomplete $3 \mathrm{D}$ object and the objects of the same class.

The current partial $3 \mathrm{D}$ object retrieval approaches can be principally categorized as: i) part based, ii) image based, and iii) bag of visual words based approaches.

\section{A. Part based approaches}

Part based approaches are based on the theory that human being examines the semantics of objects' parts to recognize it. In fact, they rely on the assumption that similar $3 \mathrm{D}$ objects consist of similar segments. Arhid et al. [9] proposed a part based approach by segmenting each $3 \mathrm{D}$ object into its constituent segments, and then for each part a descriptor is computed by the approach based on a multi-criteria employing data envelopment analysis (DEA). In order to calculate the similarity between $3 \mathrm{D}$ objects, the authors applied a new technique to compare resemblances between descriptors of 3D object's parts. Agathos et al. [10] introduced a novel retrieval approach for articulated objects. The proposed method is composed of a segmentation step, which produces the 3D object's Attributed Relation Graph (ARG), and then the Earth Movers Distance (EMD) is used to measure the similarity between two ARGs. Tierny et al. [11] took power of Reeb graph theory to ameliorate both the 3D object description and comparison procedures. Indeed, the authors represented each $3 \mathrm{D}$ object by a Reeb graph linked with geometrical signatures. The similarity measured between two $3 \mathrm{D}$ objects is examined by calculating an alternative of their maximum communal sub-graph.

\section{B. 2D image based approaches}

2D image based approaches try to represent the $3 \mathrm{D}$ object by a set of $2 \mathrm{D}$ images, and then use them in the indexing and retrieval process. Chen et al. [12] proposed the Light Field descriptor, which is considered one of the earliest $2 \mathrm{D}$ image based approaches. The authors used Fourier coefficients and Zernike moments to describe an ensemble of 2D images captured at the vertices of a dodecahedron. Papadakis et al. [13] presented PANORAMA descriptor. It consists of the 2D Discrete Wavelet Transform and 2D Discrete Fourier Transform computed on an ensemble of panoramic images of a $3 \mathrm{D}$ object, which is characterized by the orientation and position of the surface of the $3 \mathrm{D}$ object in space. Liu et al. [14] designed a multi-view latent variable model ( MVLVM) to have an undirected graph structure in which the $3 \mathrm{D}$ object's image set is treated as the observations from which to acquire the latent visual and spatial contexts. Furthermore, they expound the learning and conjecture process of MVLVM for 2D image based 3D object retrieval. Ouazzani taybi et al. [15] started by extracting for each $3 \mathrm{D}$ object a set of $2 \mathrm{D}$ slices corresponding to its three main axes, and then they use the K-means clustering method to select the representative ones, which transform the comparison between $3 \mathrm{D}$ objects into similarity computing between their $2 \mathrm{D}$ slices. This approach produces satisfactory results if the number of clusters is correctly chosen. Otherwise, the clustering step generates over-partition or under-partition. In order to remedy this problem the authors in [16] used a cluster validity index to adapt the number of clusters to the complexity of each $3 \mathrm{D}$ object.

\section{Bag of visual words based approaches}

The bag of visual words based approach has been effectively applied on 3D object indexing and retrieval approaches. It has exhibited fruitful applications in either geometry based or image-based approaches. Likewise, it has clear focal points in partial based approaches. The typical example of application of the bag of visual words system into image-based approach is the method of Furuya et al. [17], the authors used the bag of visual words method to encode the Scale Invariant Feature Transform (SIFT) characteristics of an ensemble of depth images of a 3D object into a histogram. Laga et al. [18] approach accomplished an outstanding per- 
formance in partial based approaches by applying the bag of visual words method to the Laplace-Beltrami spectrum characteristics of a collection of evenly sampled points on the $3 \mathrm{D}$ object's surface by projecting the geometry onto the Laplace-Beltrami operator's eigenvectors.

\section{Data mining}

Data mining, also called knowledge discovery in databases, is an important research domain in computer science, it is widely used in business (insurance, retail, banking, credit card fraud detection system), science research (medicine, astronomy, biological data analysis), and government security (detection of criminals and terrorists). One of the most important DM tasks is to find the association rules and to discover the interesting and useful patterns and relationships in large volumes of data [19].

At first, the association rule theory was widely utilized for marketing aims, but it could also be used in different domains of research such as the searching of frequent values, pairs or cooccurrences if the data set is in conformity with this research, as Hébrail et al. [20] posited. The classic method for resolving association rule issue is Apriori Algorithm proposed by Agrawal et al. [21]. In fact, the use of this algorithm in Data Mining allows the examination of the diverse feasible mixture of the items to discover likely relationships, which will be formulated as association rules. Following the original definition, the issue of association rules mining is presented as:

Let $I=\left\{\mathrm{i}_{1}, \mathrm{i}_{2}, \ldots, \mathrm{i}_{\mathrm{n}}\right\}$ be a set of $n$ binary attributes called items. Let $\mathrm{D}=\left\{\mathrm{t}_{1}, \mathrm{t}_{2}, \ldots, \mathrm{t}_{\mathrm{m}}\right\}$ be a set of transactions composing the database. Each transaction in $D$ is characterized by a unique transaction ID and contains a subset of the items in $I$. A rule is defined as an implication in the form $X \Rightarrow Y$ where $X, Y \subseteq I$ and $X \cap Y=\emptyset$. The sets of items (briefly item sets) $X$ and $Y$ are, respectively, called antecedent (left-hand side or LHS) and consequent (right-hand side or RHS) of the rule [22].

Several metrics can be used to measure the power of an association rule, the most frequently employed are support and confidence. The support is detailed as the proportion of the number of transactions included the antecedent $X$ and the consequent $Y$ in a dataset $D$. The confidence is defined as the probability of finding $Y$ in transactions under the condition that these transactions also contain $X$.

$$
\begin{aligned}
& \operatorname{support}(X \rightarrow Y)=\frac{|\{X \cup Y \subseteq T, T \in D\}|}{|D|} \\
& \operatorname{cofidence}(X \rightarrow Y)=\frac{\operatorname{support}(X \rightarrow Y)}{\operatorname{support}(X)}
\end{aligned}
$$

A rule will be accepted as an association rule, if its support and confidence satisfy a userspecified threshold (minsup and minconf).

\section{Our approach}

In this section, we introduce our method to index and retrieve $3 \mathrm{D}$ objects. The principal idea of our approach is to represent the $3 \mathrm{D}$ objects by an ensemble of $2 \mathrm{D}$ slices transforming the shape-matching issue between $3 \mathrm{D}$ objects into measuring the similarity between their $2 \mathrm{D}$ slices. Fig. 1 shows the architecture of the proposed approach. First, we start by 3D objects normalization to assure invariance under scaling, translation, and rotation. Second, for each $3 \mathrm{D}$ object, we extract an initial set of $2 \mathrm{D}$ slices corresponding to determined axes. Next, we describe each $2 \mathrm{D}$ slice by a vector of Zernike moments. Then, we represent the $2 \mathrm{D}$ slices of each $3 \mathrm{D}$ object in a transactional database. Thereafter, we use the Apriori algorithm to select from the initial set of 2D slices the most representative ones. Finally, the similarity metric is proposed to measure the similarity between the $3 \mathrm{D}$ objects' representative $2 \mathrm{D}$ slices. 


\subsection{D object normalization}

Generally, $3 \mathrm{D}$ objects are given in random positions, orientations and scales in the $3 \mathrm{D}$ space. In many feature extraction process, it is necessary to normalize the $3 \mathrm{D}$ object's orientation and size before feature extraction to guarantee a distinctive representation. Indeed, the normalization stage aims to assure that the similar 3D objects with different positions, orientations and scales can be correctly represented by almost the same feature descriptors.

Therefore, to ensure the invariance characteristics of our descriptor, which correlates with putting the $3 \mathrm{D}$ object into a canonical coordinate, we translate the $3 \mathrm{D}$ object's centre of mass to coincide with the origin. To address the scale normalization, the average distance of the surface of the $3 \mathrm{D}$ object from its centroid is equal to 1. The Principal Component Analysis (PCA) is used to achieve the rotation normalization.

\subsection{Creating the initial set of $2 \mathrm{D}$ slices}

Our approach consists of creating a set of 2D slices gotten by the intersection of an ensemble of plans with the $3 \mathrm{D}$ triangle mesh. In fact, the triangle meshes provide an efficient way to represent $3 \mathrm{D}$ objects. Characteristically, geometry, connectivity and property data are at a time used to represent a $3 \mathrm{D}$ triangle mesh. So as to create the initial set of $2 \mathrm{D}$ slices, we take the intersection of the $3 \mathrm{D}$ triangle mesh with plans equally spaced and orthogonal to the determined axes. Fig. 2 shows an example of a 3D object, at a given position, with its 2D slices corresponding to its $\mathrm{Y}$-axis using our approach.

At the outset, we take, for each Cartesian axes (X-, Y-, and Z-axis), the intersection of the normalized $3 \mathrm{D}$ object with 50 plans equally spaced and orthogonal to the axis. 


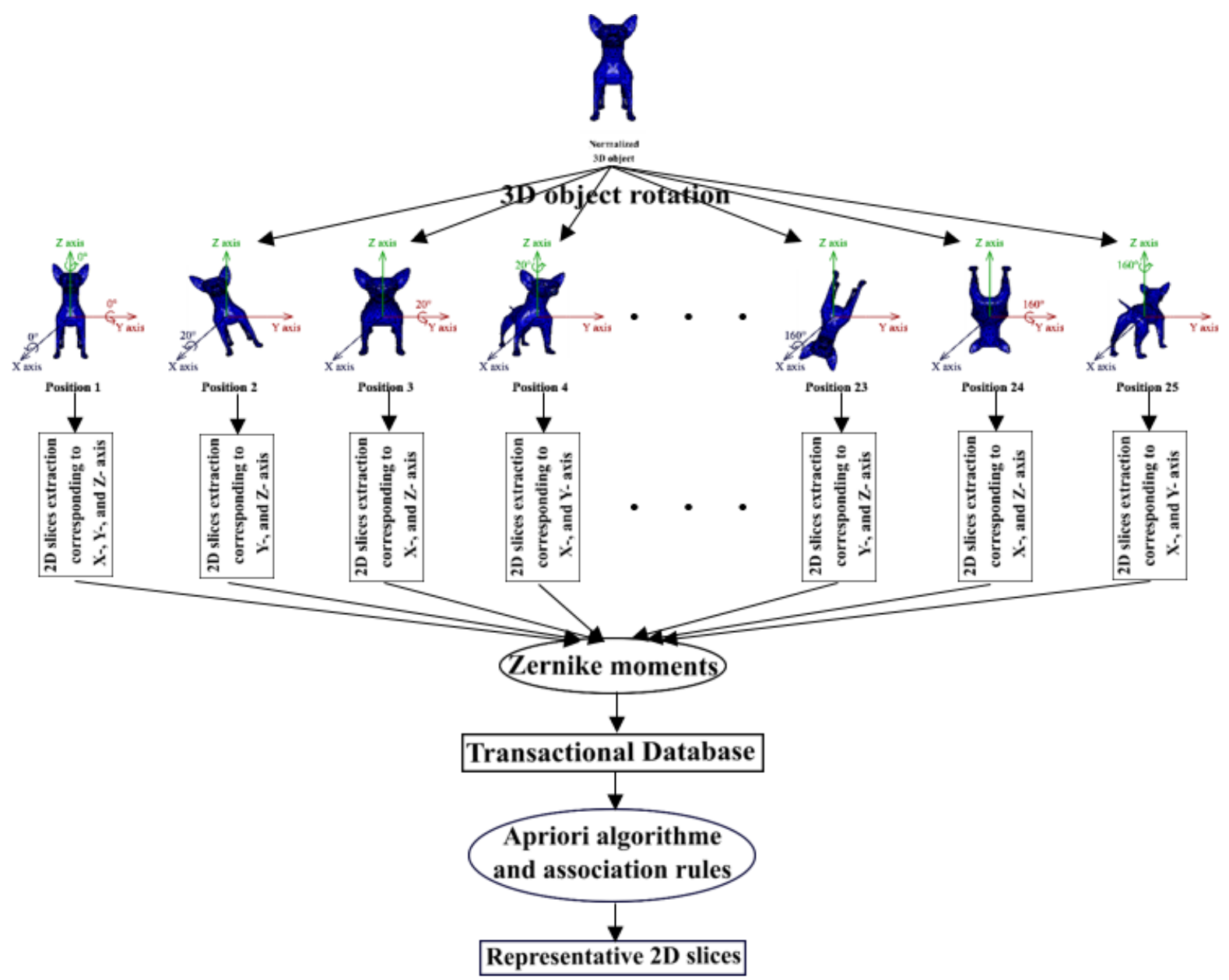

Fig. 1. The architecture of our proposed approach.

Then, we turn the $3 \mathrm{D}$ object on the three Cartesian axes (axis by axis) by $20^{\circ}$ until we reach the $160^{\circ}$. At each rotation of the $3 \mathrm{D}$ object, we capture, for only two Cartesian axes, the intersection of the turned $3 \mathrm{D}$ object with 50 plans equally spaced and orthogonal to the axis (as Fig. 1 shows); i.e. if we turned the $3 \mathrm{D}$ object on the $\mathrm{X}$-axis, we extract the $2 \mathrm{D}$ slices corresponding to the $\mathrm{Y}$ - and $\mathrm{Z}$-axis.

In fact, when we turn the $3 \mathrm{D}$ object on an axis, the slices corresponding to this axis remain the same as the slices corresponding to the same axis in the first position; there is only a rotation change. Since we will use the Zernike descriptor, which is a rotation invariant descriptor, to characterize the $2 \mathrm{D}$ slices, it is wise to eliminate the slices corresponding to the $3 \mathrm{D}$ object's rotation axis. 

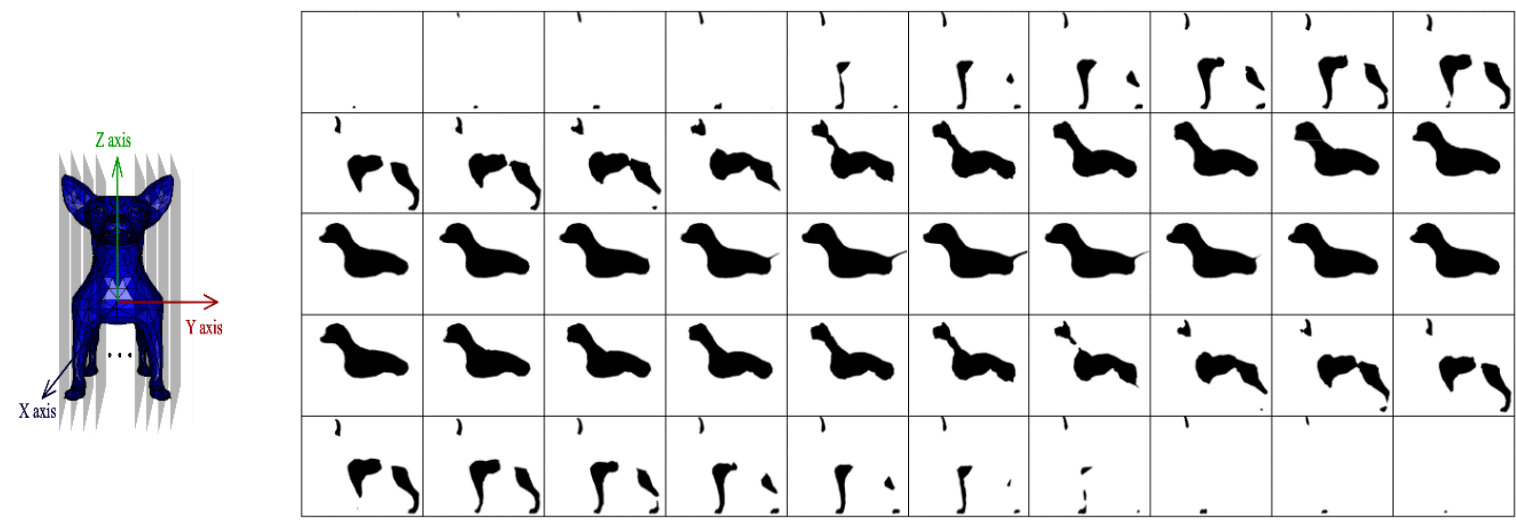

(a)

(b)

Fig. 2. Example of a $3 \mathrm{D}$ object (a) with its $2 \mathrm{D}$ slices corresponding to its $\mathrm{Y}$-axis using our approach.

\subsection{Computing the numerical signature for each $2 \mathrm{D}$ slice}

Amid the large image descriptors existing in the literature, the moments of Zernike are deemed to be the most appropriate descriptors to represent the $2 \mathrm{D}$ slices, on account of its distinctive features such as rotation invariance, small feature size, fine image representing capacity etc. Zernike moments have been successfully used in diverse image analysis and object recognition [23] [24] [25] [26]. Zhang and Lu [27] observed that the Zernike moments are very useful for capturing the main characteristics of images. This is due to the fact that these moments are orthogonal in nature, which ensures that moment values at different orders accounts for independent and unique features of an image.

In our method, only low order Zernike moments have been extracted from 2D slices. As a matter of fact, the low order Zernike moments are more able to characterize the image's gross information versus high order moments which represent the detail, and also they are fewer vulnerable to noise. As well, the magnitudes of Zernike moments' values are rotation invariant, which allows us to eliminate $2 \mathrm{D}$ slices corresponding to some axes, and better classify the extract $2 \mathrm{D}$ slices.

\subsection{Selecting the $2 \mathrm{D}$ representative slices}

Now that we have each initial 2D slice characterized by a set of Zernike Moments, we use the Apriori algorithm to select the $2 \mathrm{D}$ representative slices. To achieve this task, we represent the $2 \mathrm{D}$ slices of each $3 \mathrm{D}$ object in a transactional database. Therefore, each row (transaction) in the transactional database agrees with the 2D slices corresponding to an extraction axis.

In order to label the initial set of $2 \mathrm{D}$ slices in the transactional database, we use the cluster validity index proposed by Do et al. [28] to automatically define the optimal number of cluster according to the $3 \mathrm{D}$ object's complexity. In fact, clusters structures can have one of three states: under-partition state $\left(\mathrm{K}<\mathrm{K}^{*}\right)$, optimal partition state $\left(\mathrm{K}=\mathrm{K}^{*}\right)$ or over-partition state $\left(\mathrm{K}>\mathrm{K}^{*}\right)$. It is possible to find the optimal number of clusters using two measures: mean intra-cluster distance (MICD) and minimum inter-cluster distance (ICMD).

The MICD of the $\mathrm{i}^{\text {th }}$ cluster MICD $_{\mathrm{i}}$ is defined by:

$$
\operatorname{MICD}_{i}=\frac{1}{n_{i}} \sum_{x \in \chi_{i}}\left\|V_{i}-x\right\|
$$

Where $\chi_{i}, V_{i}$ and $n_{i}$ respectively represent the data set of the $\mathrm{i}^{\text {th }}$ cluster, the centroid of the $\mathrm{i}^{\text {th }}$ cluster and the number of data in the $i^{\text {th }}$ cluster. 


$$
\mathrm{ICMD}_{\min }=\min _{\mathrm{i} \neq \mathrm{j}} \sum_{\mathrm{x} \in \chi_{\mathrm{i}}}\left\|\mathrm{V}_{\mathrm{i}}-\mathrm{V}_{\mathrm{j}}\right\|
$$

Where $V_{i}$ and $V_{j}$ respectively represent the centroid of the $\mathrm{i}^{\text {th }}$ and $\mathrm{j}^{\text {th }}$ cluster.

Let $X=\left[x_{1}, x_{2}, \ldots, x_{n}\right]^{T}$ be a finite data set, and let $V=\left[v_{1}, v_{2}, \ldots, v_{K}\right]^{T}$ be a $K$ centroid, each $v_{i}$ characterizes one of the $K$ clusters. The under-partition measure $v_{\text {under }}(K, V, X)$ and overpartition measure $v_{\text {over }}(K, V)$, respectively defined by Eq. (5) and Eq. (6), have different scales depending on the structure and the number data. Thus, a normalization of these functions is necessary.

$$
\begin{gathered}
v_{\text {under }}(K, V, X)=\frac{1}{K} \sum_{i=1}^{K} M I C D_{i} \\
v_{\text {over }}(K, V)=\frac{K}{I C M D_{\min }}
\end{gathered}
$$

For $2 \leq \mathrm{K} \leq \mathrm{K}_{\max }$.

Let us define partition measure vectors as:

$$
\begin{gathered}
V_{\text {under }}=\left[v_{\text {under }}(2, V, X), \ldots, v_{\text {under }}\left(K_{\text {max }}, V, X\right)\right] \\
V_{\text {over }}=\left[v_{\text {over }}(2, V), \ldots, v_{\text {over }}\left(K_{\text {max }}, V\right)\right]
\end{gathered}
$$

For each vector, maximum and minimum values are computed as:

$$
\begin{gathered}
V_{\text {under_max }}=\max _{K}\left(v_{\text {under }}(K, V, X)\right) \\
V_{\text {over_max }}=\max _{K}\left(v_{\text {over }}(K, V)\right) \\
V_{\text {under_min }}=\min _{K}\left(v_{\text {under }}(K, V, X)\right) \\
V_{\text {over_min }}=\min _{K}\left(v_{\text {over }}(K, V)\right)
\end{gathered}
$$

For $K=1,2, \ldots, K_{\max }$

The normalization of each function becomes:

$$
\begin{gathered}
V_{\text {under } N}(K, V, X)=\frac{v_{\text {under }}(K, V, X)-v_{\text {under_min }}}{v_{\text {under_max }}-v_{\text {under_min }}} \\
V_{\text {overN }}(K, V)=\frac{v_{\text {over }}(K, V)-v_{\text {over_min }}}{v_{\text {over_max }}-v_{\text {over_min }}}
\end{gathered}
$$

Therefore $v_{\text {under }}$ and $v_{\text {over }}$ always lies between $\mathrm{o}$ to 1 . As a result, normalization partition measure vectors are defined as:

$$
\begin{gathered}
v_{\text {underN }}=\left[\begin{array}{c}
v_{\text {under } N}(2, V, X), \ldots \\
v_{\text {under } N}\left(K_{\text {max }}, V, X\right)
\end{array}\right]^{T} \\
v_{\text {over } N}=\left[v_{\text {over } N}(2, V), \ldots, v_{\text {over } N}\left(K_{\text {max }}, V\right)\right]^{T}
\end{gathered}
$$

The validity index, noted by $V_{S V}$, is formulated by adding $v_{\text {underN }}$ and $v_{\text {over }}$, thus is written as:

$$
V_{S V}(K, V, X)=v_{\text {under } N}(K, V, X)+v_{\text {over } N}(K, V)
$$

The optimal number of group is obtained for the smallest value of $V_{S V}(K, V, X)$ for $K$ varying from 2 to $K_{\max }$. In our method, the application of this cluster validity index, taking the inter- 
val $[2,500]$, allows us to fully determine the optimal number of cluster depending on the complexity of the 3 D object.

Now that we have determined the optimal number of cluster depending on the $3 \mathrm{D}$ object's complexity, we assign an identical label to the $2 \mathrm{D}$ slices that are in the same cluster. Next, in each row of the transactional database, we diminish the number of items (which correspond to the $2 \mathrm{D}$ slices) to the minimum by eliminating the redundancies.

To extract the representative $2 \mathrm{D}$ slices, we use the transactional database of each $3 \mathrm{D}$ object, and we apply the Apriori algorithm to extract the association rules, which will be used to determine the representative slices. After some experiments that we made to determine the suitable minsup and minconf, we conclude that it is proper to choose $25 \%$ and $90 \%$ as, respectively, minsup and minconf threshold.

\subsection{Computing the similarity}

The aim of similarity measurement is to maintain the smallest possible distances for similar objects, and to make dissimilar objects as far as possible in the feature space. Therefore, the suitable similarity measurements should be designed to compute accurately the content similarity.

In our approach, we have represented each $3 \mathrm{D}$ object by a set of characteristic slices, transforming the issue of shape-matching between $3 \mathrm{D}$ objects into how to compute the similarity between their representative 2D slices. Therefore, one-to-one correspondence was turned to many-to-many correspondence. Amongst the existing many-to-many distance measurements, Hausdorff distance has manifested its efficiency and powerful in the current retrieval works [29] [30].

Let us consider two sets $A=\left\{a_{1}, \ldots, a_{l}\right\}$ and $B=\left\{b_{1}, \ldots, b_{m}\right\}$, the Hausdorff distance $H(A, B)$ computes the level of mismatch between $A$ and $B$ by computing the distance of the point of $A$ which is furthest from any point of $B$ and vice versa. In fact, the Hausdorff pay particular attention to the dissimilarity of the two sets, but that can conduct to inappropriate results when some troubled components existed in a set. For instance, assume that all elements of A and B have a strong similarity except one pair that is different. The Hausdorff distance will ignore all similar elements by taking into consideration only the dissimilarity between the more different pair.

In order to overcome the Hausdorff weakness, we define our metric based on Hausdorff distance, and take into consideration the dissimilarity between all pairs in the set. Therefore, the dissimilarity between the $N$ representative slices of object $O$ and $M$ representative slices of query $Q$ is defined as:

$$
D(O, Q)=\max \left(\frac{\sum_{1 \leq i \leq N} i n f_{1 \leq j \leq M}\left(d_{O_{i} Q_{j}}\right)}{N}, \quad \frac{\sum_{1 \leq j \leq M} i n f_{1 \leq i \leq N}\left(d_{O_{i} Q_{j}}\right)}{M}\right)
$$

Where $d_{O_{i} Q_{j}}$ accounts for the Euclidean distance between the $i^{\text {th }}$ representative slice of $O$ and the $j^{\text {th }}$ representative slice of $Q$.

\section{Experiments and Results}

In this study, the $3 \mathrm{D}$ objects of the Princeton Shape Benchmark (PSB) database are used to evaluate our approach; this database is freely available online and widely used in many works. It contains 1814 3D objects collected from the internet, and classified by humans according to function and form. It includes a set of hierarchical classifications, separate training and test sets, annotations for each model, and a suite of software tools for generation, analysis, and visualization of shape matching results [31]. 
In order to investigate the performance of our approach, we compared it to $123 \mathrm{D}$ indexing and retrieval approaches used in the PSB (D2 Shape Distribution (D2) [32], Extended Gaussian Image (EGI) [33],Complex Extended Gaussian Image (CEGI) [34],Shape Histogram (SHELLS) [35], Shape Histogram (SECTORS)[35],Shape Histogram(SECSHEL) [35], Spherical Extent Function (EXT)[36], Radialized Spherical Extent Function (REXT) [37], Gaussian Euclidean Distance Transform (GEDT) [38], Spherical Harmonic Descriptor (SHD) [38], and Light Field Descriptor (LFD) [12]).

To objectively examine our approach, we utilized the PSB's evaluation tools with respect to the base classification. As a matter of fact, the benchmark evaluation tools generate visualizations (Precision-recall plot, Tier image, and the top five retrieval results), and statistics (Nearest neighbor (NN), First-tier (FT) and Second-tier (ST), E-Measure (EM), Discounted Cumulative Gain (DCG), and Normalized Discounted Cumulative Gain (N-DCG)) to facilitate the comparison of $3 \mathrm{D}$ object indexing and retrieval approaches. We invite the reader to consult [31], which provides more details on the evaluation criterion.

Tab. 1 summarizes the retrieval statistics for each method. LFD slightly outperformed our approach in ST (48.7\% vs. 48.6\%), and E-Measure (28.0\% vs. 27.7\%). However, our approach gives the best score in closest match metrics (NN (74.0\%), FT (39.6\%), DCG (66.8\%), and NDCG (23.5\%)), which means that our method is the best one in placing the right matches at the top of the retrieval list.

Fig. 3 shows recall-precision curves for each descriptor. As we can observe, the recallprecision curves demonstrates that our approach outperforms the compared methods and confirms the retrieval statistics shown in Tab. 1. Additionally, when the recall increases, the whole curve of our approach decreases slowly compared to the other descriptors, which means that our method is more stable.

Fig. 4 presents image visualizing nearest neighbor (white), first tier (yellow), and second tier (orange) matches using our approach in the PSB database. A strong retrieval approach should have a group of white-yellow pixels in the class-sized blocks along the diagonal. As we can notice in Fig. 4, our method has brighter pixels in the diagonal class-sized blocks showing that the $3 \mathrm{D}$ objects within the same class present higher similarity.

Fig. 5 demonstrates a portion of the retrieval $3 \mathrm{D}$ objects on the test set of the PSB using our approach. The first column in the figure shows the $3 \mathrm{D}$ objects queries and the rest of the columns present the 10 retrieved $3 \mathrm{D}$ objects in rank order. As can be found from the outcomes acquired by our method, practically all the retrieved $3^{\mathrm{D}}$ models appertain to the query object's class.

Table 1. Retrieval performances using our approach and those used in the PSB

\begin{tabular}{|c|c|c|c|c|c|c|}
\hline $\begin{array}{l}\text { Shape } \\
\text { scriptors }\end{array}$ & $\mathrm{NN}$ & FT & ST & E-Measure & DCG & N-DCG \\
\hline $\begin{array}{ll}\text { Our } & \text { ap- } \\
\text { proach } & \\
\end{array}$ & $74.0 \%$ & $39.6 \%$ & $48.6 \%$ & $27 \cdot 7 \%$ & $\underline{66.8 \%}$ & $\underline{23.5 \%}$ \\
\hline LFD & $65.7 \%$ & $38.0 \%$ & $48.7 \%$ & $28.0 \%$ & $64.3 \%$ & $18.9 \%$ \\
\hline REXT & $60.2 \%$ & $32.7 \%$ & $43.2 \%$ & $25.4 \%$ & $60.1 \%$ & $11.1 \%$ \\
\hline SHD & $55.6 \%$ & $30.9 \%$ & $41.1 \%$ & $24.1 \%$ & $58.4 \%$ & $8.0 \%$ \\
\hline GEDT & $60.3 \%$ & $31.3 \%$ & $40.7 \%$ & $23.7 \%$ & $58.4 \%$ & $8.0 \%$ \\
\hline EXT & $54.9 \%$ & $28.6 \%$ & $37.9 \%$ & $21.9 \%$ & $56.2 \%$ & $3.9 \%$ \\
\hline SECSHEL & $54.6 \%$ & $26.7 \%$ & $35.0 \%$ & $20.9 \%$ & $54.5 \%$ & $0.8 \%$ \\
\hline VOXEL & $54.0 \%$ & $26.7 \%$ & $35.3 \%$ & $20.7 \%$ & $54.3 \%$ & $0.4 \%$ \\
\hline SECTORS & $50.4 \%$ & $24.9 \%$ & $33.4 \%$ & $19.8 \%$ & $52.9 \%$ & $-2.2 \%$ \\
\hline
\end{tabular}




\begin{tabular}{|l|l|l|l|l|l|l|}
\hline CEGI & $42.0 \%$ & $21.1 \%$ & $28.7 \%$ & $17.0 \%$ & $47.9 \%$ & $-11.4 \%$ \\
\hline EGI & $37.7 \%$ & $19.7 \%$ & $27.7 \%$ & $16.5 \%$ & $47.2 \%$ & $-12.7 \%$ \\
\hline D2 & $31.1 \%$ & $15.8 \%$ & $23.5 \%$ & $13.9 \%$ & $43.4 \%$ & $-19.7 \%$ \\
\hline SHELLS & $22.7 \%$ & $11.1 \%$ & $17.3 \%$ & $10.2 \%$ & $38.6 \%$ & $-28.6 \%$ \\
\hline
\end{tabular}

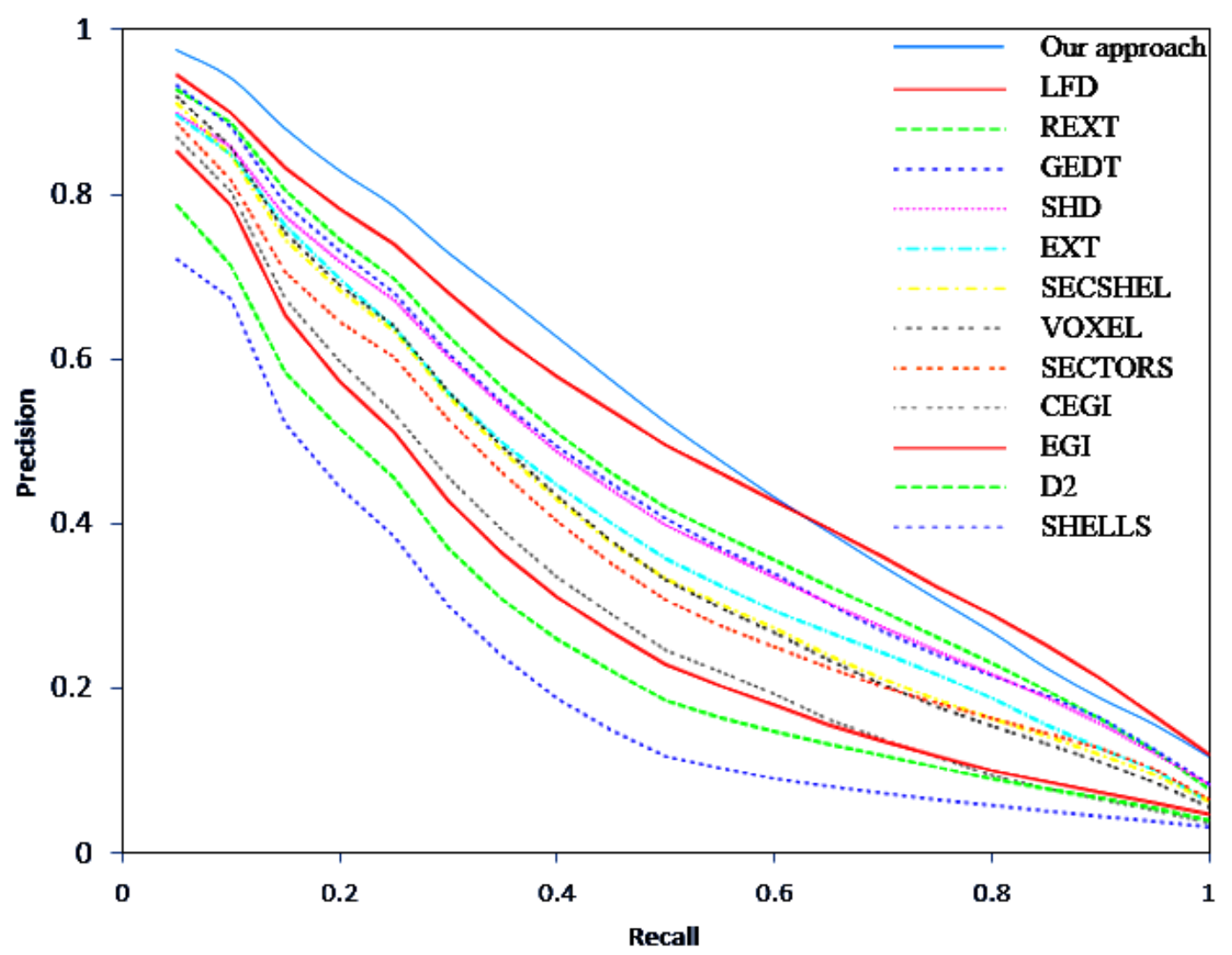

Fig. 3. Average precision-recall curves

In order to investigate the stability of our proposed method against the incomplete $3 \mathrm{D}$ objects, additional experiments have been realized. In fact, we have created a set of incomplete $3 \mathrm{D}$ objects using meshLab software by arbitrarily removing parts of $3 \mathrm{D}$ objects, and we have used them as queries. Fig. 6 demonstrates some retrieval examples using our method. The first column exposes 7 incomplete $3 \mathrm{D}$ objects queries, and each row exposes the top 10 retrieval results using our approach. From the obtained results, we can deduce that our approach performs well, and succeeds to correctly match the incomplete $3 \mathrm{D}$ objects.

\section{Conclusion}

In this paper, we introduced a new partial 3D object indexing and retrieval approach combining 2D slices and Apriori algorithm. The principal idea of our work was to take advantage of 2D slices and Data mining algorithms to upgrade the $3 \mathrm{D}$ shape description for both complete and incomplete $3 \mathrm{D}$ objects. In fact, we used the Apriori algorithm to choose from an initial set of $2 \mathrm{D}$ slices, which are extracted from the $3 \mathrm{D}$ object, the most representative ones, and then use them to describe the $3 \mathrm{D}$ object. Extensive experiments have shown that our approach gives effective results in terms of retrieval performances, and hence outperforming some of the well known methods in the literature. 


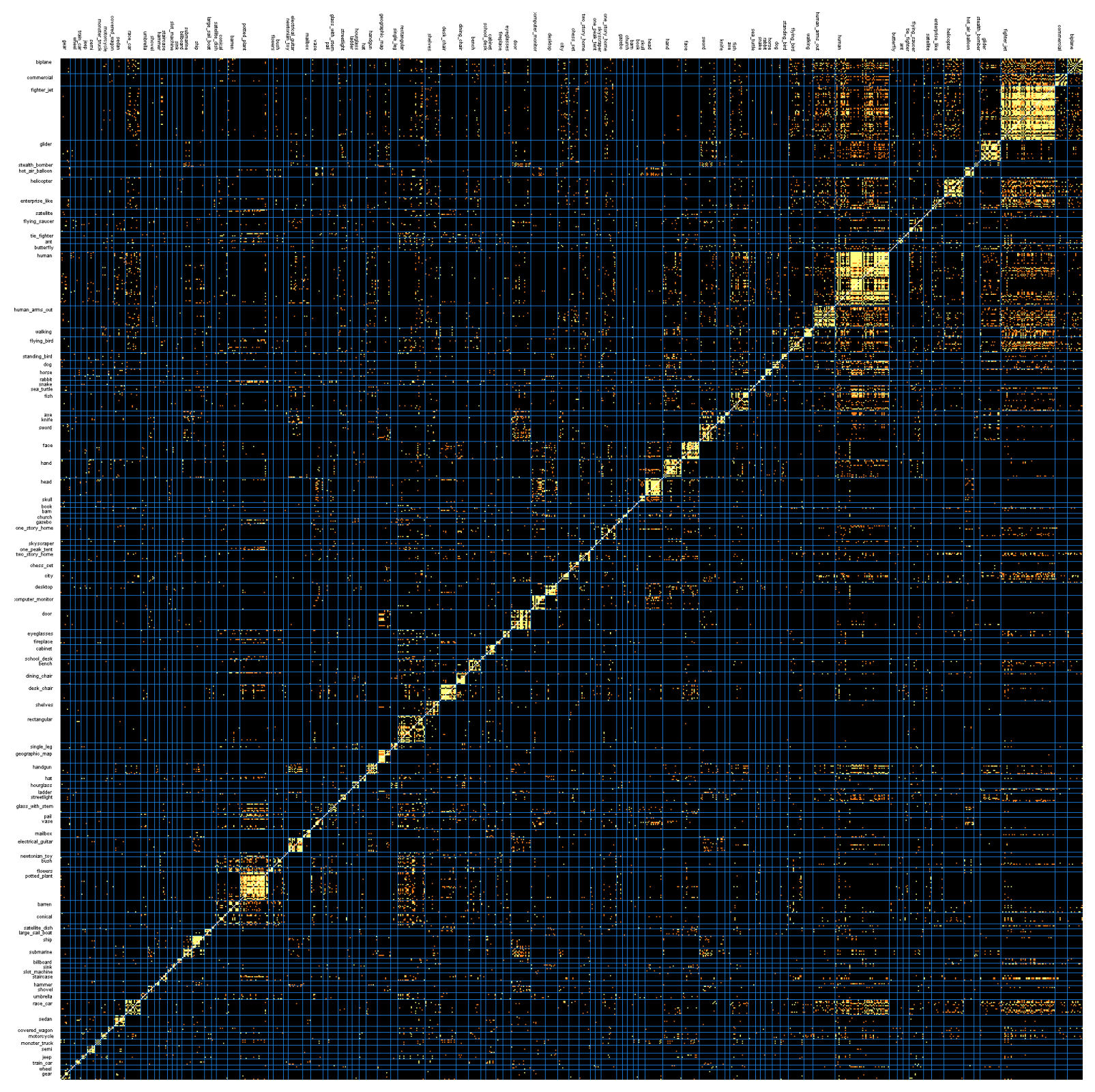

Fig .4 Tier image visualizing nearest neighbor (white), first tier (yellow), and second tier (orange) computed by matching every $3 \mathrm{D}$ object (rows) with every other $3 \mathrm{D}$ object (columns) in the PSB database using our approach. 
Query Top ten 3D objects retrieved

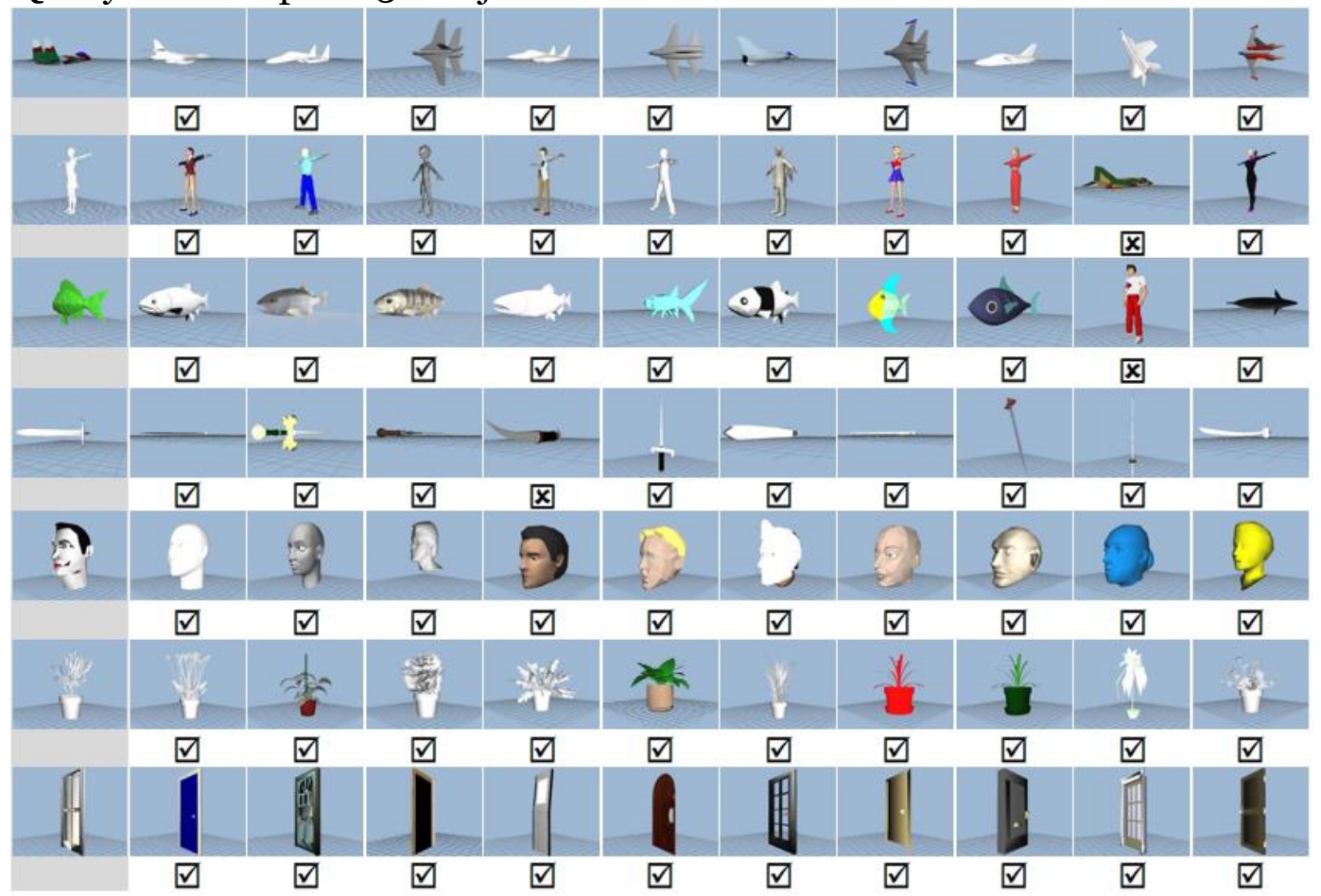

Fig. 5. Top 10 retrieved 3D objects using the proposed approach with normal query

Query Top ten 3D objects retrieved

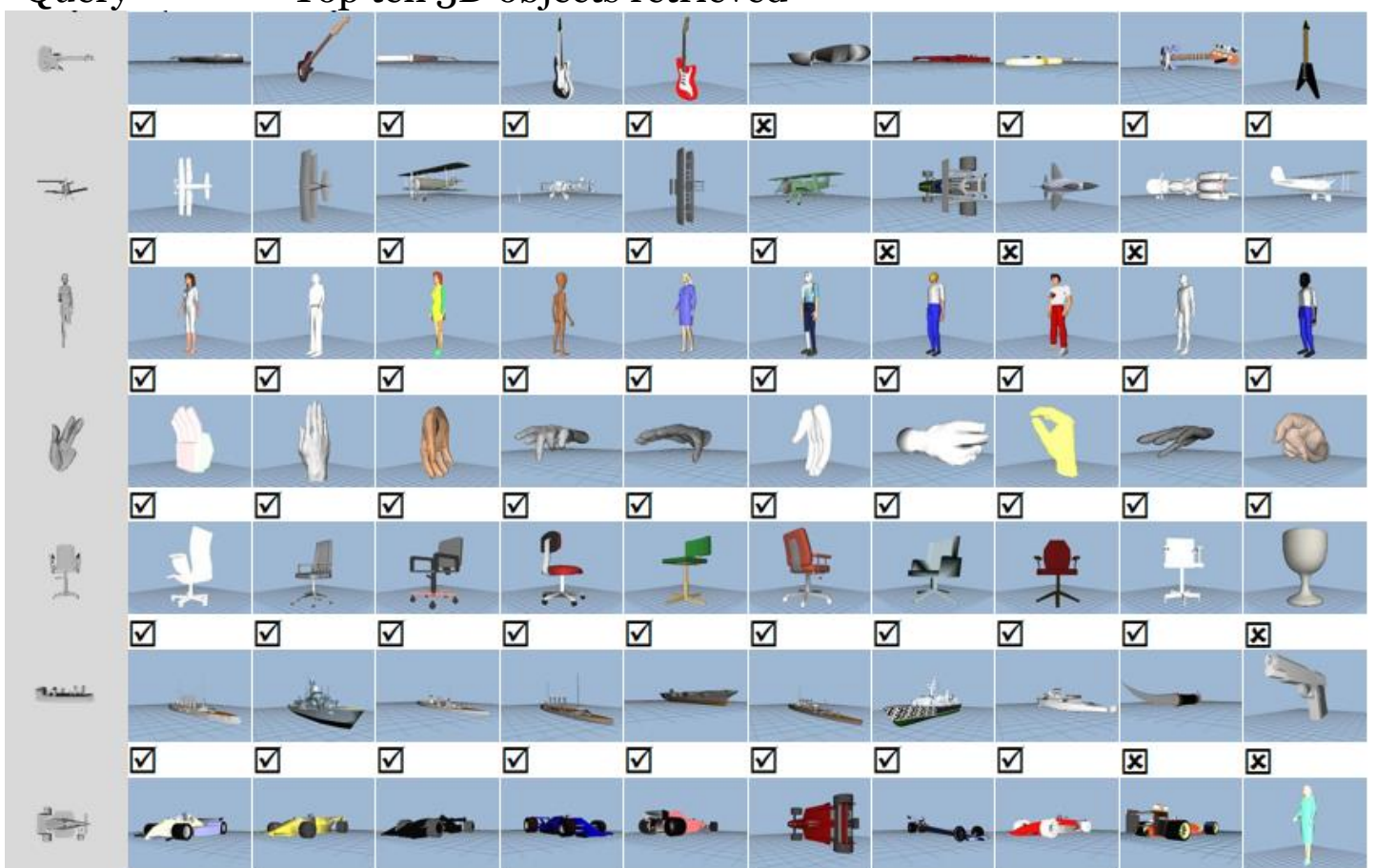

Fig. 6. Top 10 retrieved 3D objects using the proposed approach with incomplete query

In future studies, we intend to utilize a multi-agent system to make our approach less computationally expensive. Also, we will continue to adhere to partial $3 \mathrm{D}$ object retrieval approaches by taking advantage of the power of deep learning. 


\section{References}

[1] S. Zhao, L. Chen, H. Yao, Y. Zhang, and X. Sun, "Strategy for dynamic 3d depth data matching towards robust action retrieval," Neurocomputing, vol. 151, pp. 533-543, 2015.

[2] J. Cheng, W. Bian, and D. Tao, "Locally regularized sliced inverse regression based 3d hand gesture recognition on a dance robot," Information Sciences, vol. 221, pp. 274-283, 2013.

[3] G. L. Lòpez, A. P. P. Negròn, A. D. A. Jimènez, J. R. Rodrìguez, and R. I. Paredes, “Comparative analysis of shape descriptors for $3 \mathrm{~d}$ objects," Multimedia Tools and Applications, vol. 76, no. 5, pp. 6993-7040, 2017.

[4] C. Wang, M. Cheng, F. Sohel, M. Bennamoun, and J. Li, "Normalnet: A voxel-based cnn for 3d object classifi-cation and retrieval," Neurocomputing, vol. 323, pp. 139-147, 2019.

[5] M. Bouksim, k. Arhid, F. R. Zakani, M. Aboulfatah, and t. Gadi, "New approach for 3d mesh retrieval using artificial neural network and histogram of features," Scientific Visualization, vol. 10, pp. 84-94, 2018.

[6] L. Luciano and A. B. Hamza, "A global geometric framework for 3d shape retrieval using deep learning," Computers \& Graphics, vol. 79, pp. 14-23, 2019.

[7] S. Biasotti, E. M. Thompson, and M. Spagnuolo, "Context-adaptive navigation of 3d model collections," Computers \& Graphics, vol. 79, pp. 1-13, 2019.

[8] Y. Wu, H. Li, Y. Du, and Q. Cai, "Non-rigid 3d shape classification based on low-level features," in Proceedings of 2018 Chinese Intelligent Systems Conference. Springer, 2019, pp. 651-659.

[9] K. Arhid, F. R. Zakani, B. Sirbal, M. Bouksim, M. Aboulfatah, and T. Gadi, "A novel approach for partial shape matching and similarity based on data envelopment analysis,", vol. 43, no. 2, 2019.

[10] A. Agathos, I. Pratikakis, P. Papadakis, S. Perantonis, P. Azariadis, and N. S. Sapidis, "3d articulated object retrieval using a graph-based representation," The Visual Computer, vol. 26, no. 10, pp. 1301-1319, 2010.

[11] J. Tierny, J.-P. Vandeborre, and M. Daoudi, "Partial 3d shape retrieval by reeb pattern unfolding," in Computer Graphics Forum, vol. 28, no. 1. Wiley Online Library, 2009, pp. 4155 .

[12] D.-Y. Chen, X.-P. Tian, Y.-T. Shen, and M. Ouhyoung, "On visual similarity based 3d model retrieval," in Computer graphics forum, vol. 22, no. 3. Wiley Online Library, 2003, pp. 223-232.

[13] P. Papadakis, I. Pratikakis, T. Theoharis, and S. Perantonis, "Panorama: A 3d shape descriptor based on panoramic views for unsupervised 3d object retrieval," International Journal of Computer Vision, vol. 89, no. 2-3, pp. 177-192, 2010.

[14] A.-A. Liu, W.-Z. Nie, and Y.-T. Su, "3d object retrieval based on multi-view latent variable model," IEEE Transactions on Circuits and Systems for Video Technology, vol. 29, no. 3, pp. 868-880, 2018.

[15] I. Ouazzani Taybi, R. Alaoui, F. R. Zakani, K. Arhid, M. Bouksim, and T. Gadi, "A novel efficient 3d object retrieval method based on representative slices," in Multimedia Computing and Systems (ICMCS), 2016 5th International Conference on. IEEE, 2016, pp. 639-644.

[16] I. Ouazzani Taybi, M. Bouksim, R. Alaoui, and T. Gadi, "A novel partial 3d object retrieval method using adaptive slices clustering." International Journal of Intelligent Engineering and Systems, Vol.12, No.1, 2019.

[17] T. Furuya and R. Ohbuchi, "Dense sampling and fast encoding for 3 d model retrieval using bag-of-visual features," in Proceedings of the ACM international conference on image and video retrieval. ACM, 2009, p. 26. 
[18] H. Laga, T. Schreck, A. Ferreira, A. Godil, I. Pratikakis, and R. Veltkamp, "Bag of words and local spectral descriptor for 3d partial shape retrieval," in Proceedings of the Eurographics Workshop on 3D Object Retrieval (3DOR11). Citeseer, 2011, pp. 41-48.

[19] A. Dahbi, S. Jabri, Y. Ballouki, and T. Gadi, "A new method to select the interesting association rules with multiple criteria," Int. J. Intell. Eng. Syst, vol. 10, no. 5, pp. 191-200, 2017. [20] G. Hèbrail and Y. Lechevallier, "Data mining et analyse des données," Govaert G. Analyse des données. Ed. Lavoisier, Paris, pp. 323-355, 2003.

[21] R. Agrawal, R. Srikant et al., "Fast algorithms for mining association rules," in Proc. 2oth int. conf. very large data bases, VLDB, vol. 1215, 1994, pp. 487-499.

[22] G. DAngelo, S. Rampone, and F. Palmieri, "Developing a trust model for pervasive computing based on apriori association rules learning and bayesian classification," Soft Computing, vol. 21, no. 21, pp. 6297-6315, 2017.

[23] C.-W. Tan and A. Kumar, "Accurate iris recognition at a distance using stabilized iris encoding and zernike moments phase features," IEEE Transactions on Image Processing, vol. 23, no. 9, pp. 3962-3974, 2014.

[24] H. Dai and S. Liao, "Central-symmetrical property analysis on circularly orthogonal moments," Journal of Theoretical and Applied Computer Science, vol. 8, no. 2, pp. 11-26, 2014.

[25] C. Singh et al., "Improving image retrieval using combined features of hough transform and zernike moments," Optics and Lasers in Engineering, vol. 49, no. 12, pp. 1384-1396, 2011.

[26] X. Gao, Q. Wang, X. Li, D. Tao, and K. Zhang, "Zernike-moment-based image super resolution," IEEE Transactions on Image Processing, vol. 20, no. 10, pp. 2738-2747, 2011.

[27] D. Zhang and G. Lu, "Review of shape representation and description techniques," Pattern recognition, vol. 37, no. 1, pp. 1-19, 2004.

[28] K. Do-Jong, P. Yong-Woon, and P. Dong-Jo, "A novel validity index for determination of the optimal number of clusters," IEICE Transactions on Information and Systems, vol. 84, no. 2, pp. 281-285, 2001.

[29] S. Zhao, H. Yao, Y. Zhang, Y.Wang, and S. Liu, "View-based 3d object retrieval via multimodal graph learning," Signal Processing, vol. 112, pp. 110-118, 2015.

[30] Y. Gao, M. Wang, R. Ji, X. Wu, and Q. Dai, "3-d object retrieval with hausdorff distance learning," IEEE Transactions on industrial electronics, vol. 61, no. 4, pp. 2088-2098, 2013.

[31] P. Shilane, P. Min, M. Kazhdan, and T. Funkhouser, "The princeton shape benchmark," in Shape modeling applications, 2004. Proceedings. IEEE, 2004, pp. 167-178.

[32] R. Osada, T. Funkhouser, B. Chazelle, and D. Dobkin, "Matching 3d models with shape distributions," in Shape Modeling and Applications, SMI 2001 International Conference on. IEEE, 2001, pp. 154-166.

[33] B. K. P. Horn, "Extended gaussian images," Proceedings of the IEEE, vol. 72, no. 12, pp. 1671-1686, 1984.

[34] S. B. Kang and K. Ikeuchi, "Determining 3-d object pose using the complex extended gaussian image," in Proceedings. 1991 IEEE Computer Society Conference on Computer Vision and Pattern Recognition. IEEE, 1991, pp. 580-585.

[35] M. Ankerst, G. Kastenm"uller, H.-P. Kriegel, T. Seidl et al., "Nearest neighbor classification in 3d protein databases." in ISMB, vol. 99, 1999, pp. 34-43.

[36] D. Saupe and D. V. Vrani'c, "3d model retrieval with spherical harmonics and moments," in Joint Pattern Recognition Symposium. Springer, 2001, pp. 392-397.

[37] D. V. Vranic, "An improvement of rotation invariant $3 \mathrm{~d}$-shape based on functions on concentric spheres," in Proceedings 2003 International Conference on Image Processing (Cat. No. 03CH37429), vol. 3. IEEE, 2003, pp. III-757. 
[38] M. Kazhdan, T. Funkhouser, and S. Rusinkiewicz, "Rotation invariant spherical harmonic representation of 3D shape descriptors," in Symposium on geometry processing, vol. 6, 2003, pp. 156-164. 